\title{
PHOSPHOLINE IODIDE (217 M 1) (ECOTHIOPHATE IODIDE) IN THE TREATMENT OF GLAUCOMA*
}

\author{
BY \\ J. P. F. LLOYD \\ The Eye Hospital, Oxford
}

Phospholine iodide (217 M 1) (ecothiophate iodide) has the following chemical formula:

$$
\left(\mathrm{CH}_{3}\right)_{3} \cdot \stackrel{+}{\mathrm{N}} \cdot \mathrm{CH}_{2} \cdot \mathrm{CH}_{2}-\mathrm{S}-\mathrm{P}(=0)\left(\mathrm{OC}_{2} \mathrm{H}_{5}\right)_{2} \cdot \overline{\mathrm{I}}
$$

The first report of its clinical use as a miotic in the treatment of glaucoma came from Leopold, Gold, and Gold (1957). Krishna and Leopold (1959) reported their results in 110 eyes of 62 patients with glaucoma treated with it for continuous periods extending, in many cases, up to 2 years without surgical intervention. Becker, Pyle, and Drews (1959) reported its effect on the coefficient of aqueous outflow in thirty normal and 65 glaucomatous human eyes. Becker and Gage (1960) described the effects of phospholine iodide in a series of 144 eyes and of decamarium bromide in a series of 155 eyes with various forms of glaucoma, giving further details of tonographic and other results and describing the local and systemic side-effects which were encountered.

Drance and Carr (1960) reported the results of using phospholine iodide in five eyes of normal subjects and in 59 non-operated eyes showing "chronic simple" glaucoma. Later Drance reported his results in 33 eyes for periods ranging from 12 to 24 months (Drance, 1960). Pharmacological studies, especially on the impermeability of the blood-brain barrier to phospholine iodide, were reported by Koelle and Steiner (1956). Its site of action has been studied by McIsaac and Koelle (1958) and Koff and Coon (1959), and its toxicity by Schaumann and Job (1958), Mamo and Leopold (1958), and Jewell and Lehmann (1958).

The present paper attempts a detailed description of all the cases which could be traced (viz. 72 patients, 115 eyes) in which the drug was used by my two colleagues and myself at Oxford Eye Hospital during the 4 years from March, 1958, to March, 1962.

* Received for publication July 17, 1962. 


\section{Properties}

Phospholine iodide (P.I.) is available as a pure crystalline powder. It is supplied in sealed ampoules containing $6 \cdot 25,12 \cdot 5$, or $125 \mathrm{mg}$., in which it is stable indefinitely. Its solubility in water is high, and we have normally dispensed it dissolved in 0.5 per cent. chlorbutol, in 0.9 per cent. sodium chloride and water (normal saline), in strengths of $0 \cdot 1$ and 0.25 per cent. These solutions appear to remain stable for at least 4 weeks at the ambient temperatures encountered in England. Suspicion as to the potency of solutions older than this has occasionally arisen in our work and the pharmacist has therefore been instructed to ensure as far as possible that the solution should be withdrawn from patients at the end of 4 weeks and a fresh bottle dispensed. On only one occasion in our experience did a visible fungus growth appear, and this occurred within a week of dispensing.

The strengths of drop mentioned were adopted originally from the paper by Leopold, Gold, and Gold (1957), and until recently other strengths had not been investigated. A few trials in cases of open-angle glaucoma now suggest that a 0.01 per cent. solution has about the same potency as the more usual range of pilocarpine strengths ( $1-3$ per cent.), and that a 0.05 per cent. solution approximates in effect to a mixture of pilocarpine 1 per cent, and eserine $0 \cdot 12$ per cent. (c.f. Wheeler's drops-see later). We have not attempted any trials of P.I. in strengths above $0 \cdot 25$ per cent.

\section{Administration}

Frequency of administration has been governed by the twice-daily principle, though Krishna and Leopold (1959) have reported effective instillation at intervals as long as once or twice weekly. Like Drance $(1959,1960)$, I doubt whether the ordinary British patient can be relied on to adhere to such a regime. The maximum reliable interval is probably once daily, and Drance has shown that this can be effective, as in most cases the effect of one instillation lasts for 24 hours or more. Later, however, Drance appears to have reverted to twice-daily administration. Our own experience has suggested that, in some cases, the effects of an instillation may wear off in less than 24 hours. Twice-daily dosage would appear to insure against this without prolonging the tension studies which would involve a longer stay in hospital. Only occasionally have untoward effects forced us to try longer intervals and, as will be seen later, the drug usually has to be withdrawn altogether in such cases.

The effect of instillation is almost invariably an extreme miosis- $1 \mathrm{~mm}$. pupil diameter or less-which is complete within one hour. This is accompanied in most cases of glaucoma by a very marked fall in intra-ocular pressure, and in the presence of the latter a marked increase may be demonstrated in the coefficient of aqueous outflow (Becker and others, 1959). The miosis from one instillation may persist for from 7 to 27 days (Drance and Carr, 1960), and in many cases is accompanied by a very marked fall in intra-ocular pressure, beginning after about 12 hours and lasting for 3 to 5 days. With repeated instillations, many patients show a small subsequent rise in intra-ocular pressure within a week or so, but this usually remains within acceptable limits. This desirable state of affairs, once attained, may persist indefinitely. 


\section{Side-effects}

Local side-effects are common, as may well be expected with a powerful miotic, but their frequency and severity appear to compare favourably with those encountered with eserine 0.5 to 1 per cent. Initial pain or discomfort for a short time after instillation is frequent, but usually passes off after a few days; if it persists or becomes intolerable, withdrawal of the drug may become necessary. This effect combined with spasm of accommodation is naturally very marked in younger patients, below the age of, say, 55 years, and we have found the use of P.I. in such cases unrewarding except as an emergency or temporary measure.

Blurring of vision due to extreme miosis may be troublesome; the patient may become habituated to this in the course of a week or two but, especially in the presence of lens opacities, this blurring often necessitates withdrawal of the drug. The transience of such side-effects is borne out by the frequency with which longterm tolerance was achieved. We did not attempt to confirm the finding of Drance (1960) that Neosynephrine is useful in counteracting this miosis.

In one of our younger patients (aged 47) ectropion uveae with apparent cyst formation occurred after 8 weeks. Drance (1960) reported this as an occasional incident.

Oedema of the lids and eczema necessitated withdrawal in a few cases, often after long periods.

We encountered no evidence whatsoever of systemic side-effects or poisoning.

This drug is stated to act as an irreversible cholinesterase inhibitor on the peripheral neuro-muscular mechanism. Its safety is enhanced by the fact that it does not pass the blood-brain barrier. It has been shown that, if systemic poisoning does occur, it can be efficiently counteracted by pyridine aldoxine methiodide or atropine, or a combination of the two (Miller and Tainter, 1944; Schild, 1957; Jewell and Lehman, 1958; Lehman and Nicholls, 1960; Lehman, Fitch, Bloch, Jewell, and Nicholls, 1960).

\section{Experience of use of Phospholine Iodide}

In order to show the place that P.I. has come to take in my treatment of glaucoma, it is necessary briefly to consider the course of action which I have come to adopt in these cases over the last 14 years. While I have been preoccupied with deferring or avoiding surgery in open-angle glaucoma wherever possible, my two colleagues have not always agreed with me. One of them tends to try P.I. only in desperate post-operative cases, and the other pursues an intermediate course. The indications on which they have used P.I. have been so different from those used in my firm, that I have thought it best to present their cases apart from my own.

In 1948, I started taking as many of my glaucoma patients as possible into hospital for 4-hourly tension readings as an index of the stabilization which could be achieved by their current or, if indicated, an alternative treatment. At this time 4 per cent. pilocarpine was the most powerful miotic commonly used, though eserine at various strengths was occasionally tried either postoperatively or in cases in which operation was refused or impracticable. I 
found the tolerance of Eserine to be usually extremely poor, and became anxious to find an alternative. Wheeler (1944) had suggested that a mixture of pilocarpine 1 to 2 per cent., eserine 0.12 per cent., and cocaine 0.25 per cent. was better tolerated; I adopted this mixture under the title of "Wheeler's drops" as a measure intermediate between pilocarpine and surgery with some considerable success, and the compound is in use in my firm under this name to this day. Callahan (1957) reported that some of the disadvantages and dangers of di-isopropyl-fluorophosphate (D.F.P.) as a long-term miotic had been met by its use in a strength of 0.01 per cent. (as opposed to $0 \cdot 1$ per cent.) and this I rapidly and successfully adopted as a further intermediate method of treatment. In 1957, therefore, in cases of open-angle glaucoma, the sequence of measures available for the control of tension had come to be (1) Pilocarpine, (2) Wheeler's drops, (3) D.F.P. 0.01 per cent., (4) D.F.P. with Diamox added, (5) Drainage operation.

P.I. was made available to me early in 1958 . At first $I$ thought it might have a place in this series between D.F.P. and surgery, but I soon realized that its advantages over D.F.P. were many (stability in solution and water solubility especially) and that it could not only almost completely supplant D.F.P., but was occasionally rather more powerful in its effects with a superior level of tolerance.

P.I. has, therefore, been in use in my firm for just 4 years, and I hope a useful purpose will be served by the following survey of the results in the 72 cases (115 eyes) which I have been able to trace (56 personal cases and sixteen cases treated by my colleagues).

Material (Table I).-The largest group comprises 31 cases of open-angle glaucoma ( 24 of my own and 7 of my colleagues). There were also twelve doubtful open-angle cases, fourteen of angle-closure glaucoma, eight of aphakic glaucoma, four of glaucoma capsulare, and one each of juvenile, thrombotic, and secondary glaucoma.

TABLE I

72 CASES (115 EYES) INCLUDED IN THIS SERIES

\begin{tabular}{c|l|c|c|c|c|c|c}
\hline \multirow{2}{*}{ Group } & \multirow{2}{*}{$\begin{array}{c}\text { Diagnosis of } \\
\text { Glaucoma }\end{array}$} & \multicolumn{2}{|c|}{ Treated by Author } & \multicolumn{2}{|c|}{ Treated by Colleagues } & \multicolumn{2}{|c}{ Total } \\
\cline { 3 - 8 } & & Cases & Eyes & Cases & Eyes & Cases & Eyes \\
\hline A & Open-angle & 24 & 42 & 7 & 12 & 31 & 54 \\
B & Doubtful & 8 & 15 & 4 & 8 & 12 & 23 \\
C & Angle-closure & 13 & 20 & 1 & 2 & 14 & 22 \\
D & Aphakic & 6 & 6 & 2 & 2 & 8 & 8 \\
E & Capsulare & 4 & 4 & 0 & 0 & 4 & 4 \\
F & Juvenile & 0 & 0 & 1 & 2 & 1 & 2 \\
G & Thrombotic & 1 & 1 & 0 & 0 & 1 & 1 \\
H & Secondary & 0 & 0 & 1 & 1 & 1 & 1 \\
\hline \multicolumn{2}{r}{ Total } & 56 & 88 & 16 & 27 & 72 & 115 \\
\hline
\end{tabular}


(A) Open-Angle Glaucoma.-The history and progress of my own 24 cases are shown in as much detail as possible. In eighteen cases both eyes were treated and in six only one eye, so that it is clearer to describe the results in terms of eyes rather than patients. The age and sex of these patients is shown in Table II.

TABLE II

AGE, SEX, AND NO. OF EYES TREATED IN 24 PERSONAL CASES OF OPEN-ANGLE GLAUCOMA

\begin{tabular}{|c|c|c|c|c|c|c|c|}
\hline \multicolumn{2}{|c|}{ No. of Cases } & \multirow{3}{*}{ No. of Eyes } & \multicolumn{2}{|c|}{ Sex } & \multicolumn{3}{|c|}{ Age (yrs) in December, 1961} \\
\hline & & & \multirow{2}{*}{$\begin{array}{c}\text { Male } \\
9 \\
4\end{array}$} & \multirow{2}{*}{$\begin{array}{c}\text { Female } \\
9 \\
2\end{array}$} & \multirow{2}{*}{$\begin{array}{c}\text { Max. } \\
93 \\
83\end{array}$} & \multirow{2}{*}{$\begin{array}{c}\text { Min. } \\
47 \\
54\end{array}$} & \multirow{2}{*}{$\begin{array}{c}\text { Average } \\
71 \\
71\end{array}$} \\
\hline $\begin{array}{l}\text { Both Eyes Treated } \\
\text { One Eye Treated }\end{array}$ & $\begin{array}{r}18 \\
6\end{array}$ & & & & & & \\
\hline Total & 24 & 42 & 13 & 11 & 93 & 47 & 71 \\
\hline
\end{tabular}

Their history and progress is summarized in Table III (overleaf) with the treatment given immediately before P.I. was started, the number of weeks during which P.I. was given, and the results of subsequent treatment in cases in which P.I. was withdrawn.

The survey of treatment previously given revealed some interesting points. My notions expressed above of serial miotic regimes (i.e. Pilocarpine, Wheeler's drops, D.F.P., P.I.) were found in practice to have been honoured as frequently in the breach as in the execution. Thus I should have expected to find that nearly all would have been promoted to P.I. either from D.F.P. or from Wheeler's drops. In fact, this occurred in only 21 eyes; twelve were transferred straight from various strengths of pilocarpine and seven from various dosages of eserine, and two had had no previous miotic treatment at all.

The results of P.I. treatment are classified under the headings "stabilized", "eventual failure", and "failure", the implications of which are taken to be as follows:

Stabilized.-This relates primarily to tension.

In my experience, given adequate serial tension studies, visual deterioration is prevented as long as the recorded tension does not rise above $25 \mathrm{~mm}$. $\mathrm{Hg}$ on the standardized Schiötz tonometer. Certainly any record at or over $30 \mathrm{~mm} . \mathrm{Hg}$ would demand immediate reconsideration of the regime. All eyes shown under this heading were still being treated with P.I. at the time of review.

Failure.-This indicates cases in which surgery or other alternative treatment proved to be necessary within 6 weeks of starting P.I.

Eventual Failure.-This includes those cases which were given P.I. successfully for more than 6 weeks-often for long periods-before an alternative treatment became necessary for some reason. 
TABLE III

RESULTS IN 24 PERSONAL CASES OF OPEN-ANGLE GLAUCOMA (42 EYES)

\begin{tabular}{|c|c|c|c|c|c|c|c|c|c|c|c|}
\hline \multirow[b]{2}{*}{$\begin{array}{c}\text { Previous } \\
\text { Treatment }\end{array}$} & \multirow[b]{2}{*}{$\begin{array}{c}\text { No. } \\
\text { of } \\
\text { Eyes }\end{array}$} & \multicolumn{2}{|c|}{ Stabilized } & \multicolumn{2}{|c|}{$\begin{array}{c}\text { Eventual } \\
\text { Failure after } \\
6 \text { weeks }\end{array}$} & \multicolumn{2}{|c|}{$\begin{array}{l}\text { Failure in } \\
6 \text { weeks } \\
\text { or Less }\end{array}$} & \multicolumn{2}{|c|}{$\begin{array}{c}\text { Reason for } \\
\text { Failure and } \\
\text { Withdrawal of } \\
\text { P.I. }\end{array}$} & \multicolumn{2}{|c|}{ Subsequent History of "Failures" } \\
\hline & & $\mid \begin{array}{c}\text { No. } \\
\text { of } \\
\text { Eyes }\end{array}$ & $\begin{array}{c}\text { Mean } \\
\text { Dura- } \\
\text { tion } \\
\text { of P.I. } \\
\text { (wks) } \\
\text { Treat- } \\
\text { ment }\end{array}$ & $\begin{array}{c}\text { No. } \\
\text { of } \\
\text { Eyes }\end{array} \mid$ & $\begin{array}{c}\text { Mean } \\
\text { Dura- } \\
\text { tion } \\
\text { of P.I. } \\
\text { (wks) } \\
\text { Treat- } \\
\text { ment }\end{array}$ & $\begin{array}{c}\text { No. } \\
\text { of } \\
\text { Eyes }\end{array}$ & $\begin{array}{l}\text { Mean } \\
\text { Dura- } \\
\text { tion } \\
\text { of P.I. } \\
\text { (wks) } \\
\text { Treat- } \\
\text { ment }\end{array}$ & $\begin{array}{l}\text { Intoler- } \\
\text { ance of } \\
\text { Drug }\end{array}$ & $\begin{array}{c}\text { Inade- } \\
\text { quate } \\
\text { Control }\end{array}$ & Treatment & Results \\
\hline $\begin{array}{l}\text { Uncontrolled } \\
\text { by Pilo- } \\
\text { carpine } 1 \\
\text { to } 4 \text { per } \\
\text { cent. }\end{array}$ & 12 & 8 & 52 & 1 & 10 & 3 & $2 \cdot 5$ & 2 & $\left|\begin{array}{c}2 \\
\text { (one } \\
\text { with } \\
\text { Diamox) }\end{array}\right|$ & $\begin{array}{l}\text { † } 1 \text { Pilocarpine } \\
4 \text { per cent. } \\
\ddagger 1 \text { Iridencleisis } \\
\ddagger 2 \text { Trephine }\end{array}$ & $\begin{array}{l}\text { Stabilized } \\
\text { Uncontrolled } \\
\text { Stabilized }\end{array}$ \\
\hline \multirow{2}{*}{$\begin{array}{l}\text { Uncontrolled } \\
\text { by Pilo- } \\
\text { carpine with } \\
\text { Eserine or } \\
\text { Wheeler's } \\
\text { drops }\end{array}$} & \multirow[t]{2}{*}{11} & \multirow[t]{2}{*}{3} & \multirow[t]{2}{*}{127} & \multirow[t]{2}{*}{4} & \multirow[t]{2}{*}{66} & \multirow[t]{2}{*}{4} & \multirow[t]{2}{*}{3} & \multirow[t]{2}{*}{1} & \multirow[t]{2}{*}{7} & $\begin{array}{l}\text { \$ } 4 \text { Trephine } \\
3 \text { Iridencleisis }\end{array}$ & All stabilized \\
\hline & & & & & & & & & & 1 Abandoned & \\
\hline $\begin{array}{l}\text { Uncontrolled } \\
\text { by Eserine } \\
0.5 \text { to } 1 \text { per } \\
\text { cent. (One } \\
\text { case had } \\
\text { Diamox) } \\
\end{array}$ & 7 & - & - & 4 & 11 & 3 & 3 & 2 & 5 & $\begin{array}{l}3 \text { Trephine } \\
4 \text { Iridencleisis }\end{array}$ & All stabilized \\
\hline \multirow{2}{*}{$\begin{array}{l}\text { Uncontrolled } \\
\text { by DFP } \\
0.01 \text { per } \\
\text { cent. (two } \\
\text { had } \\
\text { Diamox) }\end{array}$} & \multirow[t]{2}{*}{6} & \multirow[t]{2}{*}{1} & \multirow[t]{2}{*}{193} & \multirow[t]{2}{*}{5} & \multirow[t]{2}{*}{71} & \multirow[t]{2}{*}{ - } & \multirow[t]{2}{*}{ - } & \multirow[t]{2}{*}{3} & \multirow[t]{2}{*}{2} & 3 Trephine & All stabilized \\
\hline & & & & & & & & & & 2 Abandoned & - \\
\hline $\begin{array}{l}\text { Controlled } \\
\text { by DFP } \\
0 \cdot 01 \text { per cent }\end{array}$ & 4 & 3 & 186 & 1 & 138 & - & - & 1 & - & Lens extraction & Stabilized \\
\hline $\begin{array}{l}\text { Uncontrolled } \\
\text { No treat- } \\
\text { ment }\end{array}$ & 2 & 2 & 86 & - & - & - & - & - & 一 & - & 一 \\
\hline Total & 42 & 17 & $\begin{array}{l}\text { (Min. } \\
27 \\
\text { Max. } \\
193 \text { ) } \\
\text { Mean } \\
96^{*}\end{array}$ & 15 & $\begin{array}{l}\text { (Min. } \\
8 \\
\text { Max } \\
186) \\
\text { Mean } \\
41^{*}\end{array}$ & 10 & $\begin{array}{l}\text { (Min. } \\
\frac{1}{2} \\
\text { Max. } \\
6 \text { ) } \\
\text { Mean } \\
2 \frac{1}{2}\end{array}$ & 9 & 16 & $\begin{array}{l}22 \text { Treated } \\
3 \text { Abandoned }\end{array}$ & 21 Stabilized \\
\hline
\end{tabular}

$\uparrow$ Visual difficulty later found to be due to retinal thrombosis.

\pm P.I. 0.1 per cent. restored one year after operation.

If One of these was a diabetic with retinopathy.

$\$$ One patient aged 47 developed ectropion uveae.

* (Min. 8 Max. 193) Mean 70.

The duration of treatment with P.I. is shown as the average number of weeks for the eyes in each of these groups.

The eyes included under "Failure" and "Eventual Failure" are reclassified in the following columns of Table III to show the reason for withdrawal of P.I. In some instances there was intolerance (symptomatic, visual, or allergic) of the drug and in others the ocular tension was not adequately controlled. The alternative adopted was usually some form of surgery. A few eyes were "abandoned" because surgery was not worthwhile on visual grounds, or impracticable on general grounds, or refused by the patient. 
Summary of Results.-Seventeen of the 42 eyes (over one-third) were satisfactorily stabilized on P.I. There is a suggestion here that the cases "promoted" directly from pilocarpine did rather better than the rest, and this may be worthy of further investigation.

A further fifteen eyes were stabilized for a time on P.I. (some of them for long periods) before becoming uncontrolled.

Thus 32 of the 42 eyes showed a response to P.I. of varying degrees of usefulness and durability. The stabilized group includes tolerance periods up to 193 weeks, eleven being over 100 weeks. In one "single eye" case, lid oedema and eczema developed after 186 weeks.

The seven cases (12 eyes) treated by my colleagues are summarized in Table IV, which shows each case separately with previous treatment, duration of treatment with P.I., and results.

TABLE IV

PARTICULARS OF SEVEN CASES OF OPEN-ANGLE GLAUCOMA TREATED BY THE AUTHOR'S COLLEAGUES

\begin{tabular}{|c|c|c|c|c|c|}
\hline $\begin{array}{l}\text { Case } \\
\text { No. }\end{array}$ & $\begin{array}{c}\text { Eyes } \\
\text { Treated }\end{array}$ & Previous Treatment & $\begin{array}{l}\text { Duration } \\
\text { of P.I. } \\
\text { Treatment } \\
\text { (wks) }\end{array}$ & Result & Remarks \\
\hline 1 & Both & $\begin{array}{l}\text { Uncontrolled by } \\
\text { trephine and Pilo- } \\
\text { carpine } 1 \text { per cent. }\end{array}$ & 3 & Failure & Reason not stated \\
\hline 2 & Both & $\begin{array}{l}\text { Uncontrolled by } \\
\text { Pilocarpine } 2 \text { per } \\
\text { cent. }\end{array}$ & 105 & Stabilized & \\
\hline 3 & One & $\begin{array}{l}\text { Uncontrolled by } \\
\text { Pilocarpine and } \\
\text { Eserine }\end{array}$ & 106 & Stabilized & \\
\hline \multirow[t]{2}{*}{4} & $1 \mathrm{st}$ & $\begin{array}{l}\text { Controlled by tre- } \\
\text { phine, } 4 \text { per cent. } \\
\text { Pilocarpine, and } \\
\text { Diamox }\end{array}$ & \multirow[t]{2}{*}{7} & \multirow[t]{2}{*}{ Eventual failure } & \multirow[t]{2}{*}{$\begin{array}{l}\text { Still uncontrolled by } \\
\text { Pilocarpine } 4 \text { per } \\
\text { cent. and Diamox }\end{array}$} \\
\hline & 2nd & $\begin{array}{l}\text { Uncontrolled on } \\
\text { same therapy }\end{array}$ & & & \\
\hline \multirow[t]{2}{*}{5} & $1 \mathrm{st}$ & $\begin{array}{l}\text { Uncontrolled by } \\
\text { trephine }\end{array}$ & \multirow[t]{2}{*}{25} & \multirow[t]{2}{*}{ Stabilized } & \\
\hline & 2nd & $\begin{array}{l}\text { Uncontrolled. No } \\
\text { treatment }\end{array}$ & & & \\
\hline 6 & One & $\begin{array}{l}\text { Uncontrolled by } \\
\text { Pilocarpine } 4 \text { per } \\
\text { cent. and Eserine }\end{array}$ & 108 & Eventual failure & $\begin{array}{l}\text { Still uncontrolled by } \\
\text { P.I. and Diamox } \\
\text { Operation refused }\end{array}$ \\
\hline 7 & Both & $\begin{array}{l}\text { Uncontrolled by } \\
\text { Pilocarpine } 3 \text { per } \\
\text { cent. and Diamox }\end{array}$ & 27 & Stabilized & \\
\hline
\end{tabular}


(B) Glaucoma of Doubtful Type (probably Open-angle).-The history of my eight cases in this group (15 eyes) is summarized in Table V. Four eyes had had drainage operations, and five (marked N.A.) subsequently had iris inclusion operations performed in spite of adequate control by P.I. on

TABLE V

GLAUCOMA OF DOUBTFUL TYPE (PROBABLY OPEN-ANGLE)

PARTICULARS OF EIGHT PERSONAL CASES (15 EYES) AND FOUR CASES (8 EYES) TREATED BY THE AUTHOR'S COLLEAGUES

\begin{tabular}{|c|c|c|c|c|c|c|}
\hline $\begin{array}{l}\text { In the } \\
\text { Care of }\end{array}$ & $\begin{array}{l}\text { Case } \\
\text { No. }\end{array}$ & $\begin{array}{l}\text { Eyes } \\
\text { Treated }\end{array}$ & Previous Treatment & $\begin{array}{c}\text { Dura- } \\
\text { tion } \\
\text { of P.I. } \\
\text { Treat- } \\
\text { ment } \\
\text { (wks) }\end{array}$ & Result & Remarks \\
\hline \multirow{12}{*}{ Author } & 1 & One & $\begin{array}{l}\text { Uncontrolled by } \\
\text { Pilocarpine } 2 \text { per } \\
\text { cent. }\end{array}$ & 13 & $\begin{array}{l}\text { Stabilized by } \\
\text { adding } \\
\text { Diamox }\end{array}$ & Refused operation \\
\hline & 2 & Both & $\begin{array}{l}\text { Uncontrolled by } \\
\text { Pilocarpine } 3 \text { per } \\
\text { cent. and Diamox }\end{array}$ & 32 & Stabilized & \\
\hline & \multirow[t]{2}{*}{3} & $1 \mathrm{st}$ & $\begin{array}{l}\text { Uncontrolled by } \\
\text { Wheeler's drops, } \\
\text { Diamox, iriden- } \\
\text { cleisis, and trephine }\end{array}$ & 160 & Stabilized & \\
\hline & & 2 nd & $\begin{array}{l}\text { Uncontrolled by } \\
\text { Wheeler's drops } \\
\text { and Diamox }\end{array}$ & 44 & $\begin{array}{l}\text { Eventual } \\
\text { failure }\end{array}$ & N.A. \\
\hline & 4 & Both & $\begin{array}{l}\text { Uncontrolled by } \\
\text { iridencleisis and } \\
\text { Pilocarpine } 1 \text { per } \\
\text { cent. }\end{array}$ & 29 & Stabilized & \\
\hline & \multirow[t]{2}{*}{5} & $1 \mathrm{st}$ & $\begin{array}{l}\text { Uncontrolled by } \\
\text { trephine (twice) and } \\
\text { Wheeler's drops }\end{array}$ & 138 & Stabilized & \\
\hline & & 2 nd & $\begin{array}{l}\text { Uncontrolled by } \\
\text { trephine }\end{array}$ & 8 & Stabilized & \\
\hline & 6 & Both & $\begin{array}{l}\text { Stabilized by DFP, } \\
\text { but drug not } \\
\text { tolerated }\end{array}$ & 103 & Stabilized & \\
\hline & \multirow[t]{2}{*}{7} & \multirow[t]{2}{*}{ Both } & \multirow[t]{2}{*}{$\begin{array}{l}\text { Uncontrolled by } \\
\text { DFP }\end{array}$} & 9 & $\begin{array}{l}\text { Eventual } \\
\text { failure }\end{array}$ & N.A. \\
\hline & & & & 4 & Failure & N.A. \\
\hline & \multirow[t]{2}{*}{8} & \multirow[t]{2}{*}{ Both } & \multirow{2}{*}{$\begin{array}{l}\text { Uncontrolled by } \\
\text { Pilocarpine } 3 \text { per } \\
\text { cent. }\end{array}$} & $\frac{1}{2}$ & Failure & N.A. \\
\hline & & & & 19 & $\begin{array}{l}\text { Eventual } \\
\text { failure }\end{array}$ & N.A. \\
\hline
\end{tabular}


TABLE V-continued

\begin{tabular}{|c|c|c|c|c|c|c|}
\hline $\begin{array}{l}\text { In the } \\
\text { Care of }\end{array}$ & $\begin{array}{l}\text { Case } \\
\text { No. }\end{array}$ & $\begin{array}{c}\text { Eyes } \\
\text { Treated }\end{array}$ & Previous Treatment & $\begin{array}{c}\text { Dura- } \\
\text { tion } \\
\text { of P.I. } \\
\text { Treat- } \\
\text { ment } \\
\text { (wks) }\end{array}$ & Result & Remarks \\
\hline \multirow{8}{*}{ Colleagues } & \multirow[t]{2}{*}{9} & $1 \mathrm{st}$ & $\begin{array}{l}\text { Uncontrolled by } \\
\text { Eserine } 0.5 \text { per } \\
\text { cent. }\end{array}$ & \multirow[t]{2}{*}{1 day } & Failure & \multirow[t]{2}{*}{$\begin{array}{l}\text { Still uncontrolled } \\
3 \text { yrs later by } \\
\text { iridencleisis, } \\
\text { Pilocarpine, and } \\
\text { Diamox }\end{array}$} \\
\hline & & 2nd & Stabilized by trephine & & & \\
\hline & \multirow[t]{2}{*}{10} & $1 \mathrm{st}$ & $\begin{array}{l}\text { Uncontrolled by } \\
\text { iridencleisis, } 4 \text { per } \\
\text { cent. Pilocarpine, } \\
\text { and Diamox }\end{array}$ & \multirow[t]{2}{*}{15} & \multirow[t]{2}{*}{$\begin{array}{l}\text { Eventual } \\
\text { failure }\end{array}$} & \multirow[t]{2}{*}{$\begin{array}{l}\text { Patient had dia- } \\
\text { betes } \\
\text { Later still un- } \\
\text { controlled by } \\
\text { Pilocarpine } 3 \text { per } \\
\text { cent. }\end{array}$} \\
\hline & & 2nd & $\begin{array}{l}\text { Uncontrolled by } \\
\text { trephine and lens } \\
\text { extraction }\end{array}$ & & & \\
\hline & \multirow[t]{2}{*}{11} & $1 \mathrm{st}$ & $\begin{array}{l}\text { Stabilized by Pilo- } \\
\text { carpine } 4 \text { per cent. } \\
\text { and Diamox }\end{array}$ & \multirow[t]{2}{*}{16} & \multirow[t]{2}{*}{$\begin{array}{l}\text { Eventual } \\
\text { failure with } \\
\text { Diamox }\end{array}$} & $\begin{array}{l}\text { Later stabilized by } \\
\text { Pilocarpine } 4 \\
\text { per cent. }\end{array}$ \\
\hline & & 2nd & $\begin{array}{l}\text { Uncontrolled by } \\
\text { Pilocarpine } 4 \text { per } \\
\text { cent. and Diamox }\end{array}$ & & & ? Gross neglect \\
\hline & \multirow[t]{2}{*}{12} & $1 \mathrm{st}$ & $\begin{array}{l}\text { Uncontrolled by } \\
\text { iridencleisis, Pilo- } \\
\text { carpine } 4 \text { per cent., } \\
\text { and Diamox }\end{array}$ & \multirow[t]{2}{*}{78} & \multirow[t]{2}{*}{$\begin{array}{l}\text { Stabilized by } \\
\text { adding } \\
\text { Diamox }\end{array}$} & \\
\hline & & 2nd & $\begin{array}{l}\text { Uncontrolled by } \\
\text { Pilocarpine } 4 \text { per } \\
\text { cent. and Diamox }\end{array}$ & & & \\
\hline
\end{tabular}

N.A. $=$ Suspicion of angle-closure element so strong that operation considered imperative.

the ground that their angles were so narrow gonioscopically that an element of angle-closure could not be excluded. Nevertheless, the Table shows that P.I. had a marked beneficial effect in ten eyes and gave prolonged relief in two others.

The four cases ( 8 eyes) in this group treated by my colleagues are also shown in Table V. Only two of these eyes (in one patient) were successfully stabilized with P.I.

(C) Angle-Closure Glaucoma.-My thirteen personal cases (20 eyes) and one case ( 2 eyes) treated by a colleague are summarized in Table VI. 
Five of the 22 eyes which had undergone one or more operations were controlled by P.I., three of them for 100 weeks or more.

TABLE VI

ANGLE-CLOSURE GLAUCOMA

PARTICULARS OF 13 PERSONAL CASES (20 EYES) AND 1 CASE (2 EYES) TREATED BY A COLLEAGUE

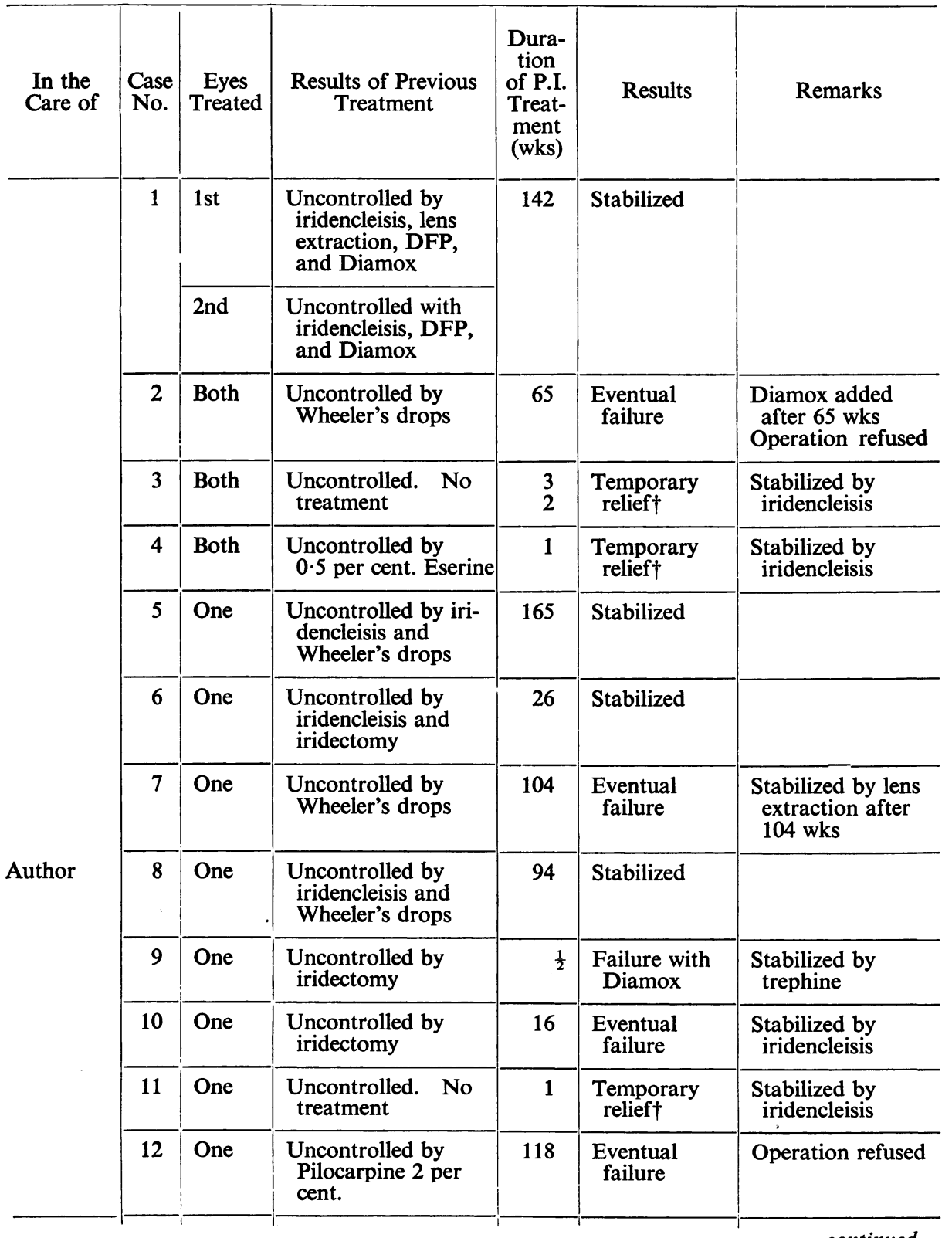


TABLE VI-continued

\begin{tabular}{|c|c|c|c|c|c|c|}
\hline $\begin{array}{l}\text { In the } \\
\text { Care of }\end{array}$ & $\begin{array}{l}\text { Case } \\
\text { No. }\end{array}$ & $\begin{array}{c}\text { Eyes } \\
\text { Treated }\end{array}$ & $\begin{array}{l}\text { Results of Previous } \\
\text { Treatment }\end{array}$ & $\begin{array}{l}\text { Dura- } \\
\text { tion } \\
\text { of P.I. } \\
\text { Treat- } \\
\text { ment } \\
\text { (wks) }\end{array}$ & Results & Remarks \\
\hline & \multirow[t]{4}{*}{$13^{*}$} & (i) $1 \mathrm{st}$ & \multirow{2}{*}{$\begin{array}{l}\text { Uncontrolled by } \\
\text { Eserine } 1 \text { per cent., } \\
\text { and Diamox }\end{array}$} & \multirow[t]{2}{*}{1} & \multirow[t]{2}{*}{$\begin{array}{l}\text { Temporary } \\
\text { relief } \dagger\end{array}$} & $\begin{array}{l}\text { Iridectomy failed } \\
\text { later (see } i i)\end{array}$ \\
\hline & & 2nd & & & & $\begin{array}{l}\text { Stabilized by } \\
\text { iridectomy }\end{array}$ \\
\hline & & (ii) One & $\begin{array}{l}\text { Uncontrolled by } \\
\text { iridectomy }\end{array}$ & 8 & $\begin{array}{l}\text { Eventual } \\
\text { failure }\end{array}$ & $\begin{array}{l}\text { Drug not tolerated } \\
\text { Lenticular myopia } \\
\text { Iridencleisis failed } \\
\text { later (see iii) }\end{array}$ \\
\hline & & (iii) One & $\begin{array}{l}\text { Uncontrolled by } \\
\text { iridencleisis and } \\
\text { Wheeler's drops }\end{array}$ & 2 days & Failure & $\begin{array}{l}\text { Stabilized by tre- } \\
\text { phine }\end{array}$ \\
\hline \multirow[t]{2}{*}{ Colleague } & 14 & $1 \mathrm{st}$ & $\begin{array}{c}\text { Stabilized. No } \\
\text { treatment }\end{array}$ & 5 & Failure & $\begin{array}{l}\text { Drug intended as } \\
\text { prophylactic but } \\
\text { ciliary cramp } \\
\text { ensued } \\
\text { Stabilized by Pilo- } \\
\text { carpine } 1 \text { per cent. }\end{array}$ \\
\hline & & 2nd & $\begin{array}{l}\text { Uncontrolled. No } \\
\text { treatment }\end{array}$ & 7 & $\begin{array}{l}\text { Temporary } \\
\text { relief } \dagger\end{array}$ & $\begin{array}{l}\text { Stabilized by } \\
\text { iridectomy }\end{array}$ \\
\hline
\end{tabular}

* This case is described in detail in the text.

$\dagger$ P.I. used as "tiding over" measure between attack settling and operation.

In eight eyes P.I. was used as a successful temporary "tiding-over" measure for a short period between an acute episode and the required operation. In three eyes (Cases 2 and 12) operation was refused and P.I. was continued as the best available alternative-combined ultimately in one case with Diamox. These three provide a good example of the futility of attempting to control angle-closure glaucoma by long-term conservative measures.

In Case 7, the patient was an old lady who did not consent to a cataract extraction (which in the end proved successful) until 2 years after starting "temporary" stabilization on P.I.

In Case 14, my colleague tried P.I. as "prophylactic" in the opposite and unaffected eye of patient with acute glaucoma, but it had to be withdrawn after 5 weeks on account of pain due to ciliary cramp.

My own Case 13 is of exceptional interest.

A 54-year-old woman was admitted to hospital in September, 1958, with bilateral subacute glaucoma. The tension was stabilized on P.I. and Diamox in both eyes, and among other findings at this stage was an outflow coefficient of $0 \cdot 20$ or better in each eye. Bilateral peripheral iridectomy was accordingly done, 
and the left eye has so far given no further trouble. After 3 months, however, it became apparent that she was having further attacks in the right eye and these I attempted to control with P.I., as it was desired to postpone further surgery. The P.I. was successful for a week or two, but a high degree of visual intolerance occurred even in the presence of a clear lens, and the distance correction increased from $-3 \mathrm{D}$ sph., $-3 \mathrm{D}$ cyl. to a steady repeatedly-checked $-5.5 \mathrm{D}$ sph., -3.5 D cyl.

An iridencleisis stabilized this eye until September, 1959, when further rises in tension became apparent. The use of P.I. as a temporary measure met with precisely the same symptomatic and optical effects, but a trephine at 6 o'clock stabilized the situation and so far, after 2 years, all is well.

A similar effect was found in Case 4 in Section E (below).

(D) Aphakic Glaucoma.-Eight eyes were treated in eight patients, six by myself and two by my colleagues (Table VII).

TABLE VII

APHAKIC GLAUCOMA

PARTICULARS OF SIX PERSONAL CASES (6 EYES) AND TWO CASES (2 EYES) TREATED BY THE AUTHOR'S COLLEAGUES

\begin{tabular}{|c|c|c|c|c|c|c|}
\hline $\begin{array}{l}\text { In the } \\
\text { Care of }\end{array}$ & $\begin{array}{l}\text { Case } \\
\text { No. }\end{array}$ & $\begin{array}{c}\text { Eyes } \\
\text { Treated }\end{array}$ & $\begin{array}{l}\text { Results of Previous } \\
\text { Treatment }\end{array}$ & $\begin{array}{c}\text { Dura- } \\
\text { tion } \\
\text { of P.I. } \\
\text { Treat- } \\
\text { ment } \\
\text { (wks) }\end{array}$ & Results & Remarks \\
\hline \multirow{6}{*}{ Author } & 1 & One & $\begin{array}{c}\text { Uncontrolled. No } \\
\text { treatment }\end{array}$ & $\frac{1}{2}$ & Stabilized & $\begin{array}{l}\text { P.I. withdrawn } \\
\text { Tension remained } \\
\text { satisfactory }\end{array}$ \\
\hline & 2 & One & $\begin{array}{l}\text { Uncontrolled by } \\
\text { cyclodialysis }\end{array}$ & 2 & Failure & $\begin{array}{l}\text { Controlled but } \\
\text { further } \\
\text { cyclodialysis }\end{array}$ \\
\hline & 3 & One & $\begin{array}{l}\text { Uncontrolled. No } \\
\text { treatment }\end{array}$ & 12 & Stabilized & $\begin{array}{l}\text { Controlled without } \\
\text { further P.I. after } \\
12 \text { wks }\end{array}$ \\
\hline & 4 & One & $\begin{array}{l}\text { Uncontrolled. No } \\
\text { treatment }\end{array}$ & 30 & Stabilized & \\
\hline & 5 & One & $\begin{array}{l}\text { Uncontrolled after } \\
\text { many operations }\end{array}$ & 24 & Stabilized & \\
\hline & 6 & One & Stabilized on DFP & 34 & Stabilized & \\
\hline \multirow{2}{*}{ Colleague } & 7 & One & $\begin{array}{l}\text { Uncontrolled by } \\
\text { Pilocarpine } 3 \text { per } \\
\text { cent. }\end{array}$ & 20 & $\begin{array}{c}\text { Eventual } \\
\text { failure }\end{array}$ & $\begin{array}{l}\text { Irritation caused } \\
\text { withdrawal. } \\
\text { Uncontrolled by } \\
\text { Pilocarpine } 4 \text { per } \\
\text { cent. }\end{array}$ \\
\hline & 8 & One & $\begin{array}{l}\text { Uncontrolled by } \\
\text { Pilocarpine } 4 \text { per } \\
\text { cent. and Diamox }\end{array}$ & 4 & Failure & $\begin{array}{l}\text { Pain intolerable } \\
\text { Uncontrolled by } \\
\text { Pilocarpine } 4 \\
\text { per cent. }\end{array}$ \\
\hline
\end{tabular}


Three appear to be stable so far for periods up to 34 weeks. Two others appeared to remain stabilized even after the withdrawal of P.I. Cases 7 and 8 showed intolerance of the drug necessitating withdrawal. Case 2 was controlled, but for reasons unstated a second cyclodialysis was done.

P.I. therefore appears to have been effective in over half the cases of this type.

(E) Glaucoma Capsulare.-Four eyes were treated personally in four patients (Table VIII). In this desperate condition P.I. seems to be little more effective than any other known treatment. In Case 4, a woman aged 80, P.I. was used 12 months after iris inclusion, but lenticular myopia appeared to be produced and this disappeared within 2 weeks of withdrawing P.I.

TABLE VIII

GLAUCOMA CAPSULARE PARTICULARS OF FOUR PERSONAL CASES (4 EYES)

\begin{tabular}{c|c|c|c|c|c}
\hline \begin{tabular}{c|c|c|c} 
Case \\
No.
\end{tabular} & $\begin{array}{c}\text { Eyes } \\
\text { Treated }\end{array}$ & $\begin{array}{c}\text { Results of Previous } \\
\text { Treatment }\end{array}$ & $\begin{array}{c}\text { Duration } \\
\text { of P.I. } \\
\text { Treatment } \\
\text { (wks) }\end{array}$ & Results & Remarks \\
\hline 1 & One & $\begin{array}{l}\text { Uncontrolled by } \\
\text { trephine and } \\
\text { Wheeler's drops }\end{array}$ & 104 & Eventual failure & $\begin{array}{c}\text { Operation refused } \\
\text { Uncontrolled after } \\
104 \text { wks } \\
\text { Only just stabilized } \\
\text { by adding Diamox }\end{array}$ \\
\hline 2 & One & $\begin{array}{l}\text { Stabilized by lens } \\
\text { extraction, Pilo- } \\
\text { carpine 4 per cent., } \\
\text { and Diamox }\end{array}$ & 56 & Stabilized & Diamox withdrawn \\
\hline 3 & One & $\begin{array}{l}\text { Uncontrolled by } \\
\text { Pilocarpine 2 per } \\
\text { cent. }\end{array}$ & 7 & Eventual failure & $\begin{array}{l}\text { Uncontrolled, but } \\
\text { stabilized by } \\
\text { trephine }\end{array}$ \\
\hline$* 4$ & One & $\begin{array}{l}\text { Uncontrolled by } \\
\text { Pilocarpine 1 per } \\
\text { cent. }\end{array}$ & 20 & Eventual failure & $\begin{array}{c}\text { Iridencleisis } \\
\text { Later uncontrolled } \\
\text { with Pilocarpine 1 } \\
\text { per cent. } \\
\text { Intorerable lenticular } \\
\text { myopia }\end{array}$ \\
\hline
\end{tabular}

* See text.

(F) Juvenile Glaucoma.-A woman aged 30, treated by one of my colleagues, had bilateral glaucoma which was not controlled by iridencleisis and 3 per cent. pilocarpine. P.I. was given four times daily and not surprisingly provoked severe intolerance.

(G) Thrombotic Glaucoma.-A man aged 61 had unilateral glaucoma which was not controlled by Diamox and 3 per cent. pilocarpine. I used P.I. with success for 3 weeks but had to withdraw it on account of irritation.

(H) Secondary Glaucoma.-A man aged 56, treated by one of my colleagues, had unilateral glaucoma which was not controlled by Diamox. 
The tension was controlled by P.I. $0 \cdot 1$ per cent. twice daily and later once nightly. There was mild discomfort and pain initially.

\section{Attendance of Patients and Quantity of P.I. Dispensed in a 6-month Period}

Table IX shows that the patients on P.I. accounted for about half of those who attended more than once, and for about 40 per cent. of the total attendances at the glaucoma clinic.

TABLE IX

ATTENDANCE RECORDS, JULY TO DECEMBER, 1961

\begin{tabular}{l|c|c}
\hline \multicolumn{1}{c|}{ Glaucoma Clinic } & All Patients & P.I. Patients \\
\hline \begin{tabular}{l|l} 
One attendance \\
More than one
\end{tabular} & 207 & $\begin{array}{c}5 \text { (less than } 2 \cdot 5 \text { per cent.) } \\
47 \text { (over } 50 \text { per cent.) }\end{array}$ \\
\hline Total patients & 86 & $52^{*}$ (about 17 per cent.) \\
\hline $\begin{array}{l}\text { Total attendances } \\
\text { Average per patient }\end{array}$ & 293 & $\begin{array}{c}132 \text { (about } 40 \text { per cent.) } \\
3 \cdot 4\end{array}$ \\
\hline
\end{tabular}

* Forty personal cases and six each for two colleagues.

$\dagger$ Total outpatient attendances for all clinics, 7,600.

The amounts of the drug dispensed during this period are set out in Table X.

TABLE X

AMOUNTS OF P.I. DISPENSED

\begin{tabular}{c|c|c|c|c|c}
\hline $\begin{array}{c}\text { No. of } \\
\text { Patients }\end{array}$ & $\begin{array}{c}\text { Strength of } \\
\text { Solution } \\
\text { (per cent.) }\end{array}$ & Total Amount (oz.) & \multicolumn{2}{c}{ Amount per Patient (oz.) } \\
\hline 47 & $0 \cdot 1$ & 65 (approx. 1,700 mg.) & 4 & 0.5 & 1.33 \\
\hline 2 & 0.25 & $4 \frac{3}{8}$ (approx. 135 mg.) & 1.5 & 0.25 & 0.95 \\
\hline
\end{tabular}

In addition, three patients used both strengths during this period, and consumed $6.75 \mathrm{oz}$. between them:

\begin{tabular}{l|l|l|l}
\hline \multicolumn{2}{c|}{ Strength of Solution (per cent.) } & $0 \cdot 1$ & $0 \cdot 25$ \\
\hline \multirow{2}{*}{ Amount consumed (oz.) } & Patient A & $1 \cdot 25$ & $1 \cdot 5$ \\
& Patient B & 1 & 1 \\
& Patient C & 1 & 1 \\
\hline & Total & $3 \cdot 25$ & $3 \cdot 5$ \\
\hline
\end{tabular}

This brings the total P.I. dispensed in 6 months to just over $76 \mathrm{oz}$. 


\section{Summary and Conclusions}

(1) The literature on the use of phospholine iodide as a miotic in the treatment of glaucoma is briefly surveyed.

(2) The physical and pharmacological characteristics investigated were found to correspond closely to those described by previous authors.

(3) The method of use by the author is described and the results encountered in various types of glaucoma over a period of 4 years are surveyed.

(4) Rather fewer local side-effects were encountered than have been described by previous authors. In no case did any suspicion of systemic poisoning arise.

(5) A category of cases of open-angle glaucoma was observed which is not mentioned by previous authors. Mainly because of the longer follow-up period, many "eventual failures" were recorded which in the long run could not be controlled even by P.I. and required alternative (usually surgical) treatment. The nature and status of these cases are examined. They were not so numerous as the cases which had remained stabilized for long periods at the end of this survey.

(6) P.I. therefore offers a miotic and tension-lowering effect comparable with or better than either eserine or DFP. The tolerance of this effect appears to be substantially better with P.I. than with the other drugs. P.I. is stable in solution, being neither dangerously volatile nor as toxic as DFP, and it is water-soluble, thereby avoiding the discomfort caused by a film of oil on the cornea.

(7) P.I. has proved a powerful and safe agent for the conservative treatment especially of open-angle glaucoma, which should prove valuable in the hands of those who prefer to defer or avoid surgical treatment as far and as long as possible.

(8) In angle-closure glaucoma, P.I. is not recommended except for emergency treatment or for short-term "tiding over" before surgery. There has been said to be a risk of "paradoxical tension rise", but this phenomenon has not yet been encountered by the author.

I should like to express my gratitude to my colleagues, Mr. A. C. L. Houlton and Mr. V. B. Purvis, for permission to use their cases in the survey. My gratitude is due to the Campbell Pharmaceutical Company for a generous and continued supply of phospholine-iodide, without which this work would not have been possible. The drug is now available in the United Kingdom from Messrs. Samoore Limited, who are to undertake its distribution on behalf of the Campbell Pharmaceutical Company.

\section{REFERENCES}

Becker, B., and GaGe, T. (1960). A.M.A. Arch. Ophthal., 63, 102.

CAYle. C. C., and DREwS, R. C. (1959). Amer. J. Ophthal., 47, 635.

Callahan, A. (1957). I Ibid., 43, 281.

Drance, S. M. (1959). Trans. Canad. ophthal. Soc., 1959, 21 and 22, 84 (1960). Trans. ophthal. Soc. U.K., 80, 387. and CARR, F. (1960). Amer. J. Ophthal., 49, 470.

Jewell, H. A., and Lehman, R. A. (1958). Fed. Proc., 17, 381.

KoFf, G. Y., and CoON, J. M. (1959). Ibid., 17, 384. 
Koelle, G. B., and Steiner, E. C. (1956). J. Pharmacol. exp. Ther., 118, 420.

Krishina, N., and LeOPOLD, I. H. (1959). A.M.A. Arch. Ophthal., 62, 300.

Leman, R. A., and Nicholls, Maude E. (1960). Proc. Soc. exp. Biol. (N.Y.), 104, 550. , Fitch, H. M., Bloch, L. P., Jewell, H. A., and Nicholls, M. E. (1960). J. Pharmacol. exp. Ther., 128, 307.

Leopold, I. H., Gold, P., and Gold, D. (1957). A.M.A. Arch. Ophthal., 58, 363.

Mamo, J. G., and LeOPOLD, I. H. (1958). Amer. J. Ophthal., 46, 724.

McIsaac, R. J., and Koelle, G. B. (1958). Fed. Proc., 17, 393.

Miller, L. C., and TAINTER, M. L. (1944). Proc. Soc. exp. Biol (N. Y.), 57, 261.

Schaumann, W., and Job, C. (1958). J. Pharmacol. exp. Ther., 123, 114.

SCHILD, H. O. (1957). Pharmacol. Rev., 9, 242.

WHEELER, J. R. (1944). Trans. ophthal. Soc. U.K., 64, 274.

\section{Additional Bibliography}

BoYD, B. F. (1959-60). " "Highlights of Ophthalmology”, vol. 3, p. 295.

Coyle, J. T., Weiner, A., Frank. P., and Leonard, A. (1961). Amer. J. Ophthal., $52,867$.

HolmberG, A. "Phospholine Iodide in the Treatment of Chronic Simple Glaucoma". (In preparation).

Krishna, N., Leopold, I. H., and Lehman, R. A. (1963). Trans. Amer. ophthal. Soc. (in the press).

LAWLOR, R. C., and LEE, P. F. Read at a meeting of the Massachusetts Eye and Ear Alumni Association and the New England Ophthalmological Society on April 16, 1958, at Boston, Mass.

Osserman, K. E., Cohen, E. S., and Genkins, G. (1959). "Proc. II Int. Myasthenia Gravis Symposium, Los Angeles, 1958".

SACKS-WILNER, A., and SACKS-WILNER, E. (1961). Amer. J. Ophthal., 51, 695.

Seward, W. H., and Albert, D. G. (1958). A preliminary report read at the meeting of the Alumni Association of the Episcopal Eye, Ear, and Throat Hospital, on May 3, 1958. Washington, D.C. 